\title{
Cytomegalovirus promotes murine glioblastoma growth via pericyte recruitment and angiogenesis
}

\author{
Harald Krenzlin, ${ }^{1}$ Prajna Behera, ${ }^{1}$ Viola Lorenz, ${ }^{2}$ Carmela Passaro, ${ }^{1}$ Mykola Zdioruk, ${ }^{1}$ Michal O. Nowicki, ${ }^{1}$ Korneel Grauwet, ${ }^{1}$ \\ Hong Zhang, ${ }^{1}$ Magdalena Skubal, ${ }^{1}$ Hirotaka Ito, ${ }^{1}$ Rachel Zane, ${ }^{1}$ Michael Gutknecht, ${ }^{3}$ Marion B. Griessl, ${ }^{3}$ Franz Ricklefs, ${ }^{4}$ Lai Ding, ${ }^{5}$ \\ Sharon Peled, ${ }^{6}$ Arun Rooj, ${ }^{1}$ C. David James, ${ }^{7}$ Charles S. Cobbs, ${ }^{8}$ Charles H. Cook, ${ }^{3}$ E. Antonio Chiocca, ${ }^{1}$ and Sean E. Lawler ${ }^{1}$ \\ 'Department of Neurosurgery, Brigham and Women's Hospital, ${ }^{2}$ Division of Newborn Medicine, Boston Children's Hospital, and ${ }^{3}$ Department of Surgery, Beth Israel Deaconess Medical Center, \\ Harvard Medical School, Boston, Massachusetts, USA. ${ }^{4}$ Department of Neurosurgery, University Medical Center Hamburg-Eppendorf, Hamburg, Germany. ${ }^{5}$ Program for Interdisciplinary Neuroscience, \\ NeuroTechnology Studio, Brigham and Women's Hospital, Boston, Massachusetts, USA. Department of Radiology, Brigham and Women's Hospital, Harvard Medical School, Boston, Massachusetts, \\ USA. 'Department of Neurosurgery, Feinberg School of Medicine, Northwestern University, Chicago, Illinois, USA. ${ }^{8}$ Swedish Neuroscience Institute, Ben and Catherine Ivy Center for Advanced Brain Tumor \\ Treatment, Seattle, Washington, USA.
}

Cytomegalovirus (CMV) has been implicated in glioblastoma (CBM); however, a mechanistic connection in vivo has not been established. The purpose of this study is to characterize the effects of murine CMV (MCMV) on GBM growth in murine models. Syngeneic GBM models were established in mice perinatally infected with MCMV. We found that tumor growth was markedly enhanced in $\mathrm{MCMV}^{+}$mice, with a significant reduction in overall survival compared with that of controls $(P<0.001)$. We observed increased angiogenesis and tumor blood flow in $\mathrm{MCMV}^{+}$mice. MCMV reactivation was observed in intratumoral perivascular pericytes and tumor cells in mouse and human CBM specimens, and pericyte coverage of tumor vasculature was strikingly augmented in MCMV+ mice. We identified PDCF-D as a CMV-induced factor essential for pericyte recruitment, angiogenesis, and tumor growth. The antiviral drug cidofovir improved survival in $\mathrm{MCMV}^{+}$mice, inhibiting MCMV reactivation, PDGF-D expression, pericyte recruitment, and tumor angiogenesis. These data show that MCMV potentiates CBM growth in vivo by increased pericyte recruitment and angiogenesis due to alterations in the secretome of CMV-infected cells. Our model provides evidence for a role of CMV in CBM growth and supports the application of antiviral approaches for CBM therapy.

\section{Introduction}

Glioblastoma (GBM) is the most common malignant brain tumor, with approximately 10,000 new cases per year in the US. There is no effective treatment, and patient survival remains dismal (1, 2). The standard-of-care treatment combining maximal surgical resection, irradiation, and temozolomide has a median survival of 14.6 months (3), and 5-year survival rates are below 10\% (4). Despite remarkable progress in our understanding of the genetics and cell biology of GBM $(1,2)$, little progress has been made in improving patient survival. Major clinical challenges include tumor invasiveness and the delivery of drugs across the bloodbrain barrier as well as inter- and intratumoral heterogeneity and drug resistance. Recent work has suggested a potential role for human cytomegalovirus (HCMV) in GBM growth, although this is not well understood at present.

HCMV is highly prevalent in human populations, and following resolution of primary infection, persists for the lifetime of its host in a latent state, periodically reactivating during periods of stress and immunocompromise (5). Interestingly, HCMV proteins and nucleic acids have been identified in up to $90 \%$

Conflict of interest: The authors have declared that no conflict of interest exists Copyright: () 2019 American Society for Clinical Investigation

Submitted: July 16, 2018; Accepted: February 5, 2019.

Reference information: J Clin Invest. 2019;129(4):1671-1683.

https://doi.org/10.1172/JCl123375. of GBM specimens (6-10) as well as in some other cancers (11, 12). Accumulating clinical data support the relevance of HCMV in GBM (13-19), with some encouraging responses reported with HCMV-targeted immunotherapies (14-18). Vaccination with DCs pulsed with tumor lysates led to expansion of mostly anti-CMV $\mathrm{T}$ cells in 1 responder (14), and CMV pp65 mRNApulsed autologous DCs showed promising long-term survival data in GBM (15-17). Ex vivo expansion of anti-CMV T cells and their administration in combination with temozolomide led to increased long-term progression-free survival (18). Data from primary GBM patients suggested that the antiviral drug valganciclovir could prolong median overall survival (19).

Although various HCMV proteins increase GBM cell proliferation, invasion, and angiogenesis $(20,21)$, a mechanistic link between HCMV and cancer in vivo has not been established, and the role of HCMV in GBM remains a subject of debate, with some groups unable to detect the presence of CMV in tumor specimens $(22,23)$. The use of mouse models to investigate the role of CMV in tumor growth would therefore be helpful in understanding the potential importance of CMV in GBM as well as providing an opportunity to rationally investigate antiviral therapeutic approaches. Using a genetically engineered GBM mouse model, we previously showed that systemic murine CMV (MCMV) infection accelerates malignant glioma progression (24), but no mechanism was established. To address this question, here we developed and characterized an MCMV GBM mouse model based on 


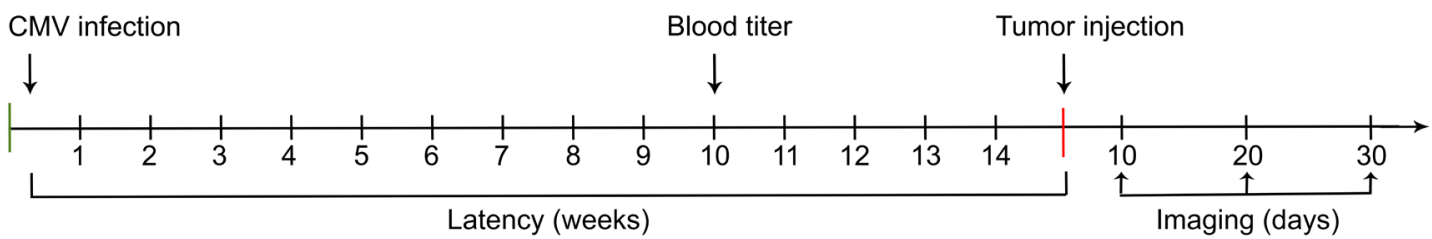

B

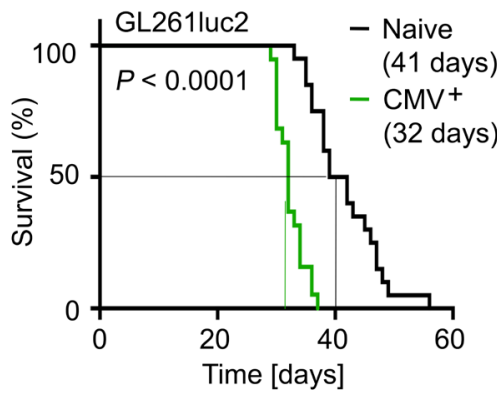

C

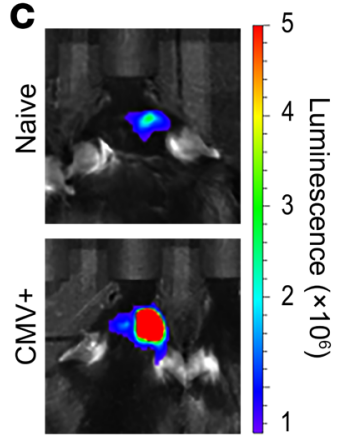

D

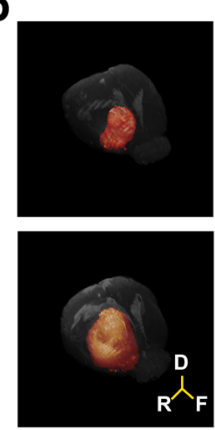

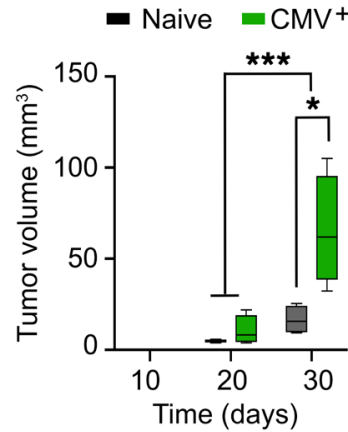

$\mathbf{E}$

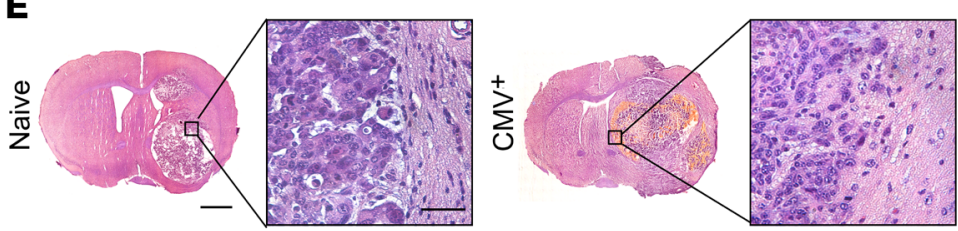

Figure 1. MCMV infection accelerates GBM growth in mice. (A) Experimental overview. (B) Kaplan-Meier curves of GL261Luc2 tumor-bearing mice. Uninfected, $n=20$; $\mathrm{MCMV}^{+}, n=19$. $P<0.0001$, log-rank test. Median survival is indicated on plot and shown in parentheses. (C) BLI and (D) MRI analysis of tumor-bearing $\mathrm{MCMV}^{+}$and control animals 30 days after tumor implantation. (D) Tumor volume rendering from MRI images (left), tumor volume over time (right). $n=3$. Box extends from the 25 th to 75 th percentile, and the median is indicated by a horizontal line. The whiskers represent the maximum and minimum values. Statistical analysis was performed by 2 -way ANOVA with Bonferroni's correction. ${ }^{*} P<0.05 ;{ }^{* *} P<0.005$. (E) H\&E staining of GL261Luc2 tumors at end points. Scale bars: $1 \mathrm{~mm}$ (left panels); $50 \mu \mathrm{m}$ (right panels).

orthotopically injected murine GBMs in a syngeneic background. Our data demonstrate that tumor growth is significantly faster in the presence of MCMV and that angiogenesis is significantly $(P<$ $0.005)$ elevated in these tumors, with a striking increase in pericyte coverage of tumor-associated blood vessels. We identified PDGF-D as an essential mediator of these effects. The angiogenic phenotype was reversed by the antiviral drug cidofovir. These data support a role for CMV in accelerating GBM growth via a proangiogenic mechanism and provide a rationale for the use of antiviral therapies in CMV-associated tumors, such as GBM.

\section{Results}

$C M V$ accelerates GBM growth in a mouse model. To investigate the role of CMV in GBM in vivo, C57BL/6 mice were infected at P2 with $\triangle \mathrm{m} 157$ Smith strain $\mathrm{MCMV}\left(\mathrm{MCMV}^{+}\right)$and allowed to resolve over at least 15 weeks (24) (Figure 1A). We stereotactically implanted luciferase-expressing murine GL261Luc2 GBM cells intracranially in $\mathrm{MCMV}^{+}$and naive control mice. $\mathrm{MCMV}^{+}$mice had significantly shorter survival than controls $(P<0.001)$ (Figure 1B) and earlier onset of clinical signs of deterioration, including weight loss (Supplemental Figure 1A; supplemental material available online with this article; https://doi.org/10.1172/JCI123375DS1). Bioluminescence imaging (BLI) revealed significantly faster tumor growth in $\mathrm{MCMV}^{+}$mice compared with controls (Figure 1C and Supplemental Figure 1B). This was confirmed by T2-weighted
MRI, which showed significantly larger tumor volumes in $\mathrm{MCMV}^{+}$ mice (Figure 1D and Supplemental Figure 1C). Histologic analysis showed increased hemorrhage and poorly defined tumor margins in $\mathrm{MCMV}^{+}$mice compared with controls (Figure 1E).

Increased angiogenesis in $M C M V^{+}$murine GBM. Further histological examination showed a pronounced increase in Ki67 and $\mathrm{CD} 31$ staining in $\mathrm{MCMV}^{+}$mice, suggesting enhanced cell proliferation and tumor angiogenesis (Figure 2, A and B). Consistent with this, image analysis (25) showed that total blood vessel length, total area of vessel coverage, and vessel branching were significantly increased in $\mathrm{MCMV}^{+}$mouse tumors (Figure 2B). We confirmed this MCMV-associated phenotype using a second murine GBM cell line, CT-2A (26), which also displayed significantly shorter survival and increased blood vessel parameters in $\mathrm{MCMV}^{+}$mice compared with controls (Figure 2, C and D). Accordingly, increased intratumoral blood flow was observed in $\mathrm{MCMV}^{+}$ mice compared with controls by arterial spin-labeling-functional MRI (ASL-fMRI) (Figure 2E). Thus, our data show that the presence of preexisting MCMV infection is associated with increased angiogenesis, elevated intratumoral blood flow, and faster tumor growth in a mouse GBM model.

Detection of CMV in pericytes and tumor cells in both mouse and human GBM. HCMV immediate early 1 (IE1) and pp65 gene products have been detected in human GBM (6-10). Similarly, we detected MCMV expression by immunofluorescence microscopy 
A
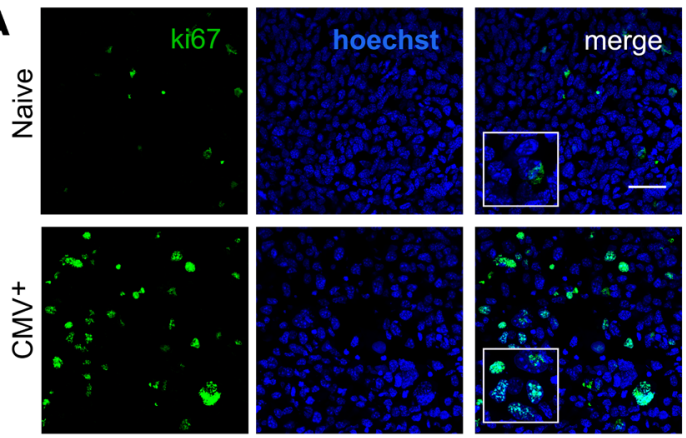

B
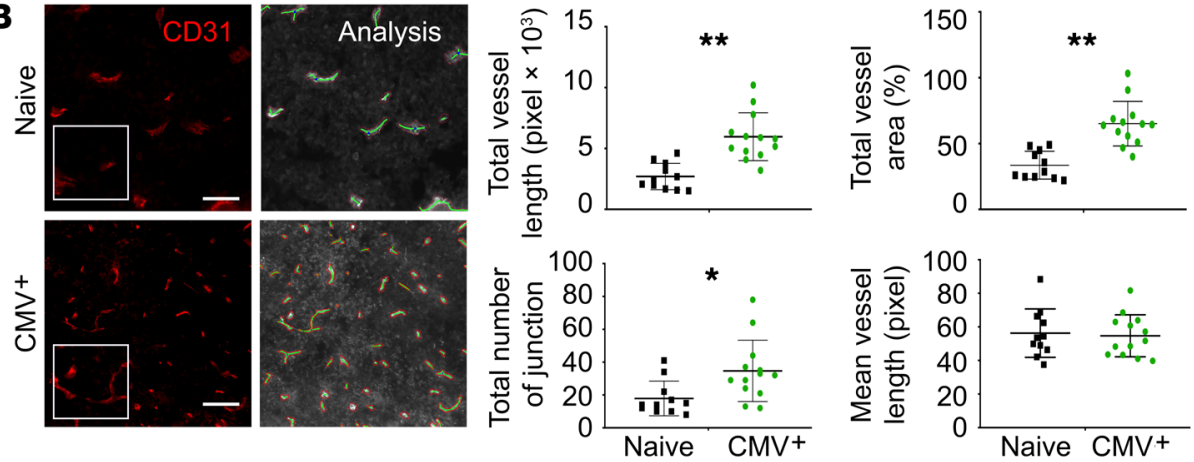

C
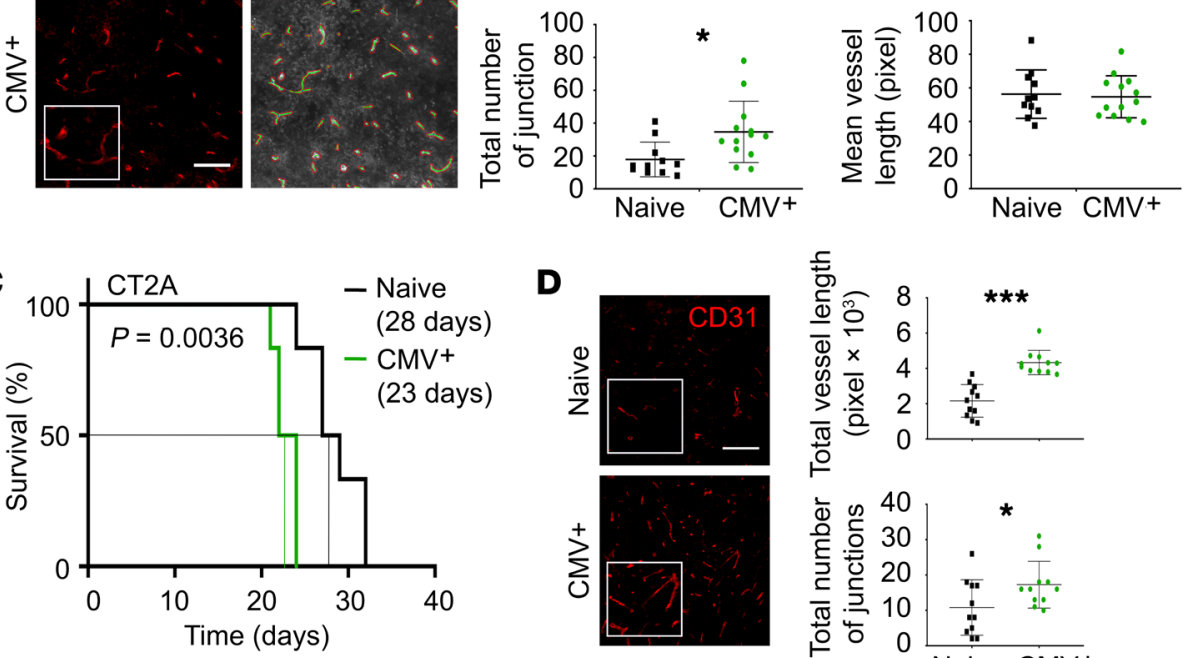

E
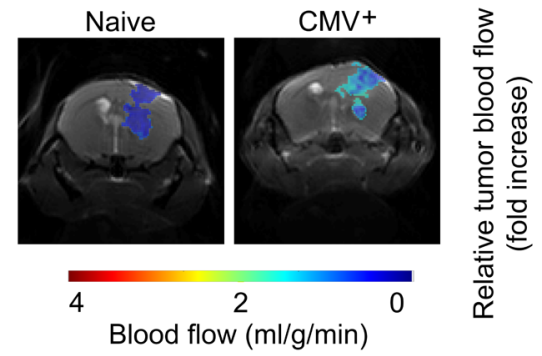
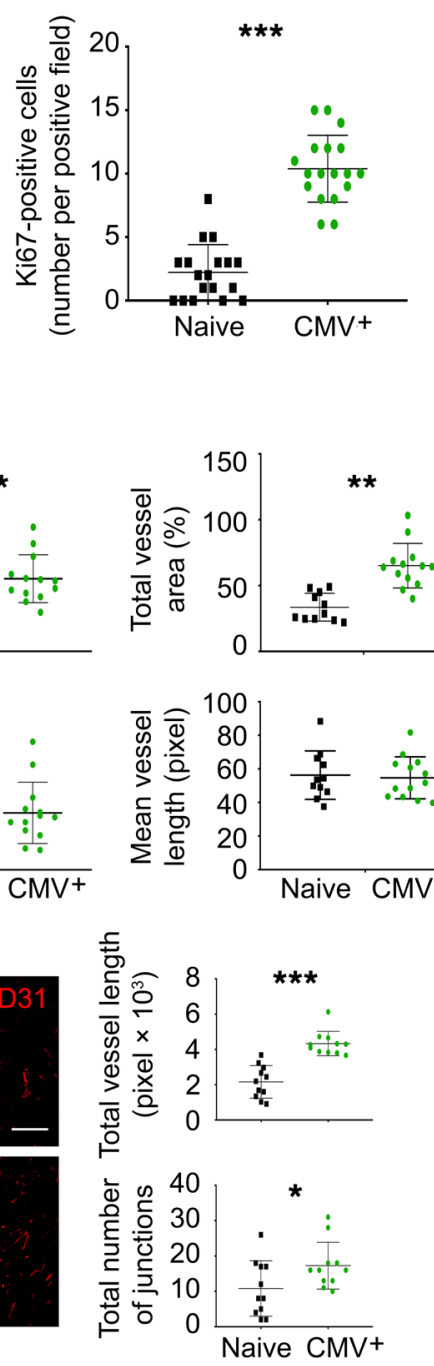

Figure 2. MCMV infection accelerates GBM blood vessel formation in mice. (A) Ki67 (green) immunofluorescence in brain sections taken from animals at the end point of survival studies. DAPI-stained nuclei are shown in blue. Scale bar: $50 \mu \mathrm{m}$. Graph shows Ki67-positive nuclei counts from 12 independent fields in 3 tumor samples. ${ }^{* *} P<0.005$, Student's $t$ test. (B) CD31 (red) immunofluorescence in sections from GL261Luc2 tumors at survival end points (left panels). Scale bars: $100 \mu \mathrm{m}$. AngioTool analysis (right panels). Graphs show data for vascular parameters. Images are from 12 independent fields for each condition. $n=4 .{ }^{*} P<0.05$; ${ }^{* *} P<0.01$ Student's $t$ test. (C) Kaplan-Meier survival curve of mice intracranially implanted with the murine CT-2A GBM cell line. Uninfected $(n=6)$ vs. $\mathrm{MCMV}^{+}(n=6) . P<0.01$, log-rank test. Median survival is indicated on plot and shown in parentheses. (D) Left panels show CD31 immunofluorescence staining (red) of tumor vasculature in 20 $\mu \mathrm{m}$ sections from CT-2A tumors in control and $\mathrm{MCMV}^{+}$animals at survival end points. Scale bar: $100 \mu \mathrm{m}$. Graphs show representations of the data for vascular parameters based on analysis of angiogenesis and vessel morphology. Images are from 12 independent fields and 3 tumor specimens for each condition. ${ }^{*} P<0.05$; ${ }^{* *} P$ $<0.005$, Student's $t$ test. (E) ASL-fMRI showing $\mathrm{T} 2$ coronal sections with ASL heatmap overlay. Quantitative analysis of both groups at corresponding ROls. $n$ $=3$. Box extends from the 25th to 75th percentile, and the median is indicated by a horizontal line. Whiskers represent the maximum and minimum values. ${ }^{*} P<$ 0.05 , Student's $t$ test. in GBMs from $\mathrm{MCMV}^{+}$but not control mice (Figure 3A and Supplemental Figure 2A) and observed a time-dependent increase of the MCMV IE1 homolog m123 mRNA by quantitative reverse-transcriptase PCR (qRT-PCR) after tumor implantation (Figure 3B). MCMV immunostaining was mostly localized in tumors and was heterogeneous. Extratumoral MCMV immunostaining was confined to the choroid plexus and ventricular regions and was otherwise absent from normal brain tissue, consistent with previous observations $(24,27)$ (Supplemental Figure 2B). No MCMV reactivation was detected in lung tissue from $\mathrm{MCMV}^{+}$tumor-bearing mice (Supplemental Figure 2C). Costaining of tumors from $\mathrm{MCMV}^{+}$ mice for the endothelial cell marker CD31 localized MCMV in the perivascular niche (Figure 3C). MCMV is known to infect pericytes (28), and immunostaining also colocalized with the pericyte marker NG2 in close proximity to tumor-associated blood vessels (Figure 3C). Additionally, MCMV colocalized with tumor cell markers CD133 and luciferase (Supplemental Figure 2D). Thus, MCMV is present in both tumor cells and the perivascular niche of GBMs in $\mathrm{MCMV}^{+}$mice.

Immunostaining for NG2 revealed striking differences between $\mathrm{MCMV}^{+}$mice and controls, with extensive coverage of tumor vasculature with $\mathrm{NG}^{+}$cells in $\mathrm{MCMV}^{+}$mice only (Figure 3D). Immunostaining and flow cytometry showed a marked increase (up to $400 \%$ ) of intratumoral $\mathrm{NG}^{+}$cells in $\mathrm{MCMV}^{+}$mice 

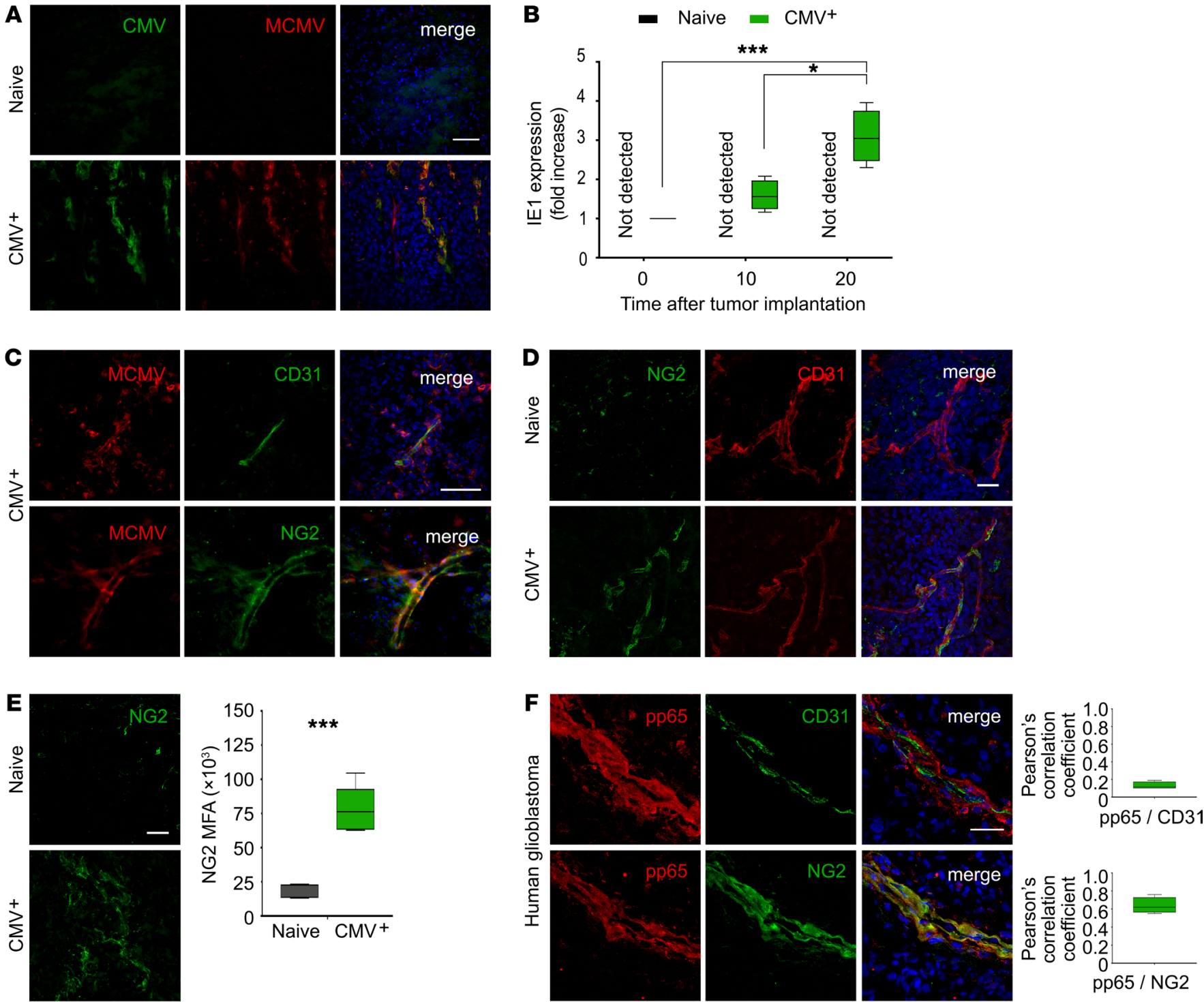

Figure 3. CMV associates with vascular pericytes in GBM. (A) CMV (green, Virusys CA150-1 antibody; red, Virusys CA003-100 antibody) immunofluorescence staining in brain sections from animals at the end point of survival studies. DAPI-stained nuclei are shown in blue. Scale bar: $50 \mu \mathrm{m}$. (B) Real-time qPCR analysis of MCMV IE1/m123 mRNA levels in GL261Luc2 tumors from MCMV+ and naive mice. $n=3$. Box extends from 25th to 75 th percentile, and median is indicated by horizontal line. Whiskers represent maximum and minimum values. ${ }^{*} P<0.05$; ${ }^{* * *} P<0.005$, Holm-Šídák test. (C) CMV (red, Virusys CA003-100), CD31 (green), and NG2 (green) immunofluorescence in tumor sections from MCMV+ mice. DAPI-stained nuclei are shown in blue. Scale bar: $50 \mu \mathrm{m}$. (D) CD31 (red), NG2 (green), and nuclei (blue) immunofluorescence in tumors from MCMV+ mice. Scale bar: $50 \mu \mathrm{m}$. (E) NG2 fluorescence intensity in 12 independent fields of view from murine GBMs. $n=3$. Box extends from 25th to 75th percentile, and median is indicated by horizontal line. Whiskers represent maximum and minimum values. Scale bar: $50 \mu \mathrm{m} .{ }^{* *} P<0.005$, Student's $t$ test. (F) CMV (red, Virusys CA003-100), CD31 (green), and NG2 (green) immunostaining immunofluorescence in human CBM. Scale bar: $50 \mu \mathrm{m}$. Pearson's rank colocalization. $n=3$. Box extends from 25 th to 75 th percentile, and median is indicated by horizontal line. Whiskers represent maximum and minimum values.

(Figure 3E and Supplemental Figure 2E). NG2 staining in $\mathrm{MCMV}^{+}$ animals strongly colocalized with the pericyte marker PDGFR- $\beta$, whereas no colocalization was observed with Olig2, a classic marker of NG2-positive glial cells (28), confirming that these MCMV/ NG2-positive cells are pericytes and not glial cells (Supplemental Figure 2F). Together, these data show MCMV-associated accumulation of pericytes in GBM vasculature and suggest that these pericytes can harbor CMV in vivo.

To confirm our findings in human GBM, we immunohistochemically visualized HCMV (Figure $3 \mathrm{~F}$ and Supplemental Fig- ure 3, A and B). HCMV pp65 and IE1 were not detected in normal human brain by immunofluorescence (Supplemental Figure 3C). In human tumor specimens, HCMV colocalized with GBM markers CD133, Olig2, and vimentin (Supplemental Figure 3, D and E). Also, strong colocalization of $\mathrm{HCMV}$ with perivascular $\mathrm{NG}^{+}$cells, but not CD $31^{+}$endothelial cells (Figure $3 \mathrm{~F}$ and Supplemental Figure 3D), was observed in human GBM, a finding consistent with HCMV association with pericytes. Furthermore, IE1 was detected in 17 out of 18 GBM samples and absent in pooled cortical control RNA (Supplemental Figure 3F). In summary, our data show wide- 
A
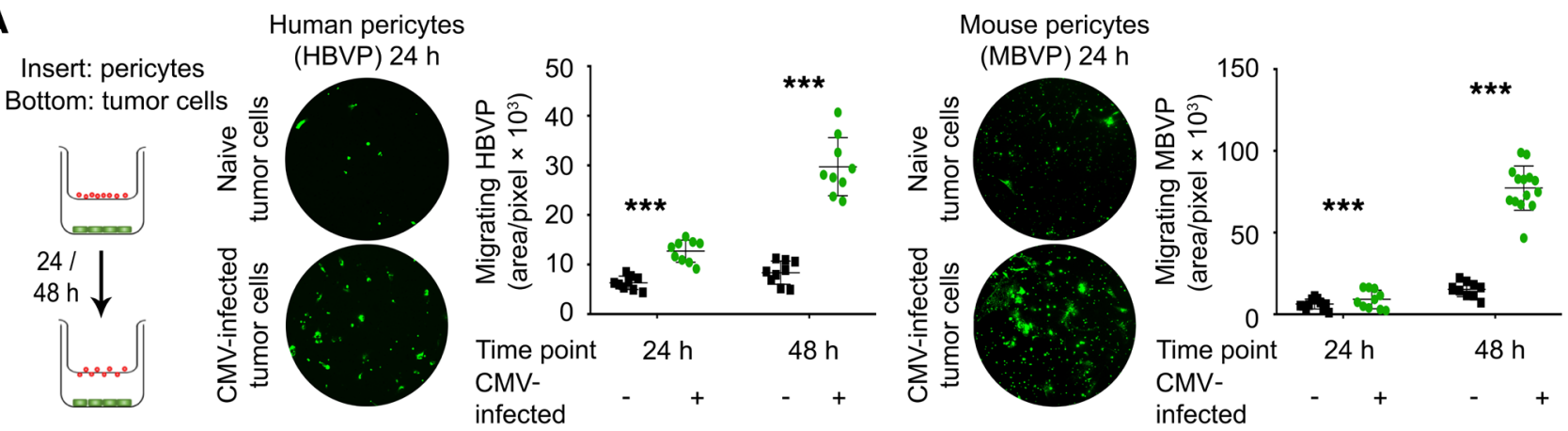

B
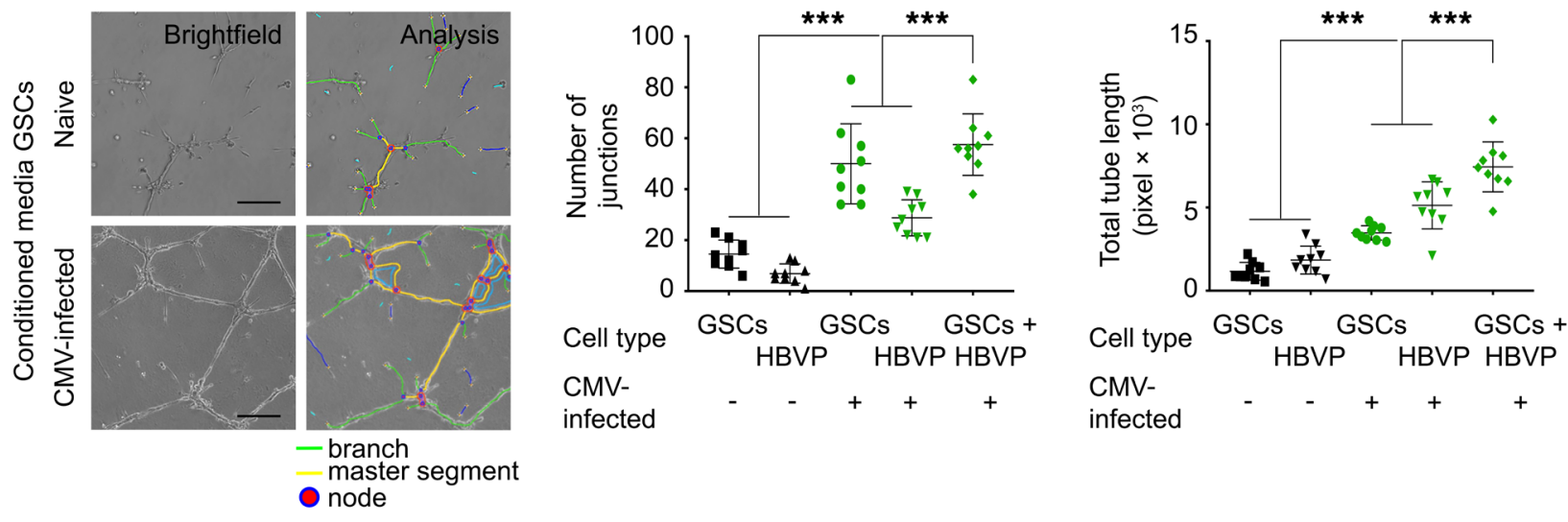

infected
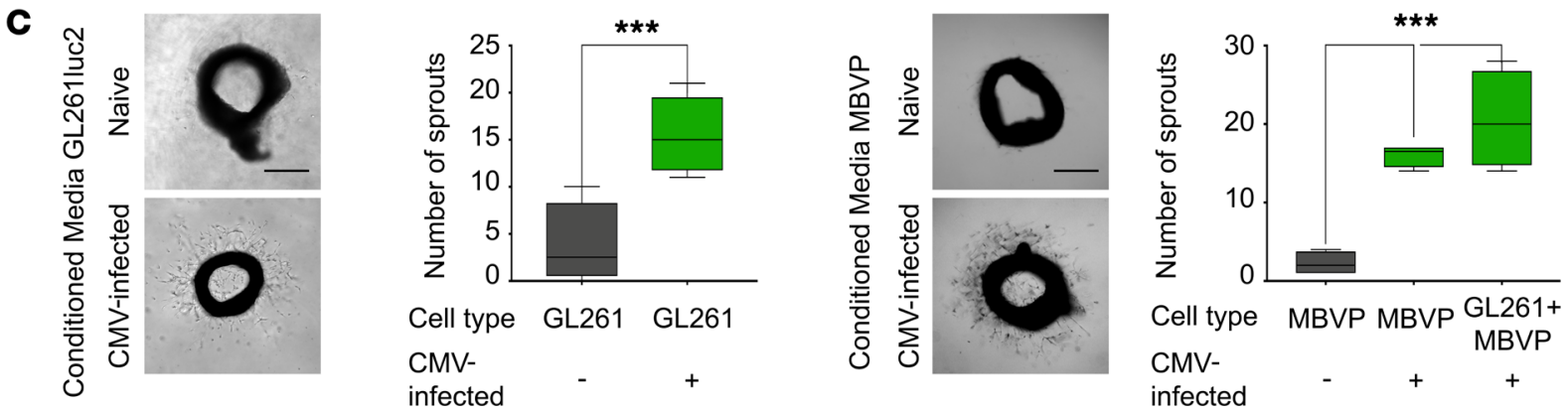

Figure 4. Conditioned medium from CMV-infected GBM cells increases pericyte migration and endothelial cell tube formation. (A) Transwell migration of HBVPs and MBVPs. Data are presented as mean \pm SD. ${ }^{* * *} P<0.005$, Holm-Šídák test. (B) HBMEC tube formation on Matrigel performed in the presence of conditioned media from HCMV-infected human GSCs or HBVPs. Data are presented as mean \pm SD. ${ }^{* *} P<0.005$, Holm-Šídák test. (C) Aortic ring assay performed using conditioned media from uninfected and CMV-infected GL261Luc2 cells or MBVPs. Graphical representation shows number of sprouting vessels. Data are presented as box and whisker plot. Box extends from 25th to 75th percentile, and median is indicated by horizontal line. Whiskers represent maximum and minimum values. ${ }^{* *} P<0.005,2$-way ANOVA. Scale bars: $50 \mu \mathrm{M}$ (B); $1 \mathrm{~mm}$ for (C).

spread detection of HCMV in GBM, and while the perivascular niche seems to be a major site of CMV infection, infected cells are observed throughout the tumor tissue in all specimens $(n=5)$ analyzed. Colocalization of NG2 and pp65 was estimated as very high $(86.2 \% \pm 11.1 \%)$. Thus, in human GBM, HCMV staining patterns are similar to those observed in the $\mathrm{MCMV}^{+}$mouse GBM model.

The CMV-infected cell secretome increases pericyte migration and angiogenesis in vitro. Pericytes have been implicated in GBM and are known to participate in angiogenesis, vessel stabilization, and regulation of cerebral blood flow (29-32). We therefore studied how CMV might mechanistically enhance pericyte accumulation and angiogenesis in GBM. Because CMV is readily detectable in both human and murine tumor cells, we hypoth- esized that CMV-infected tumor cells might attract pericytes. Using Transwell coculture assays, we confirmed that brain vascular pericytes migrate faster toward CMV-infected tumor cells compared with uninfected controls in both human and murine contexts (Figure 4A). To further investigate the effects of CMV infection of pericytes and GBM cells on angiogenesis, we cultured human brain microvascular endothelial cells (HBMECs) with conditioned media from HCMV-infected patient-derived GBM stem-like cells (GSCs) or human brain vascular pericytes (HBVPs). Both media induced significant increases in tube formation compared with control media, and when conditioned media from HCMV-infected GSCs and HBVPs were combined, tube formation was further enhanced (Figure 4B). 
A

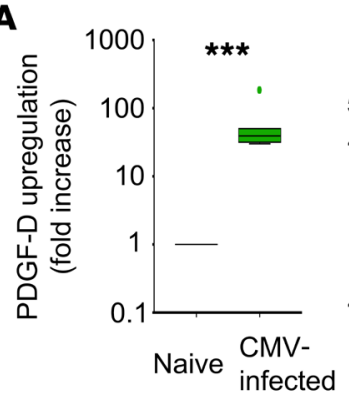

B

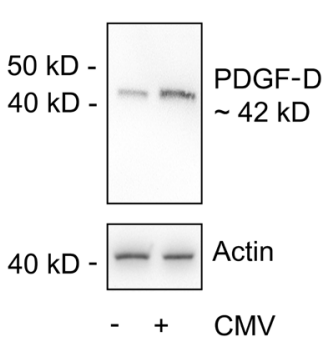

C

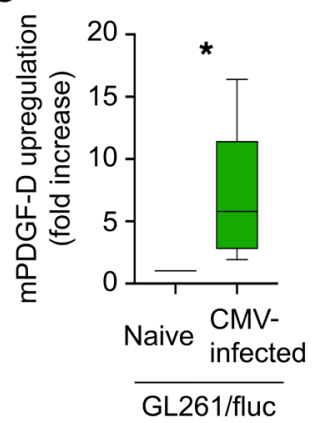

D

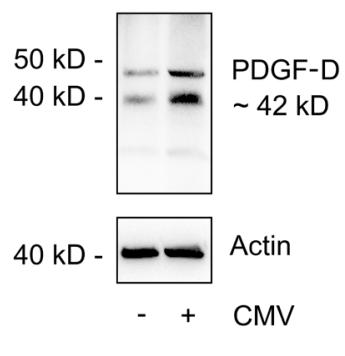

PN GSCs

$\overline{\mathrm{PN} G S C \mathrm{~s}}$

GL261/fluc
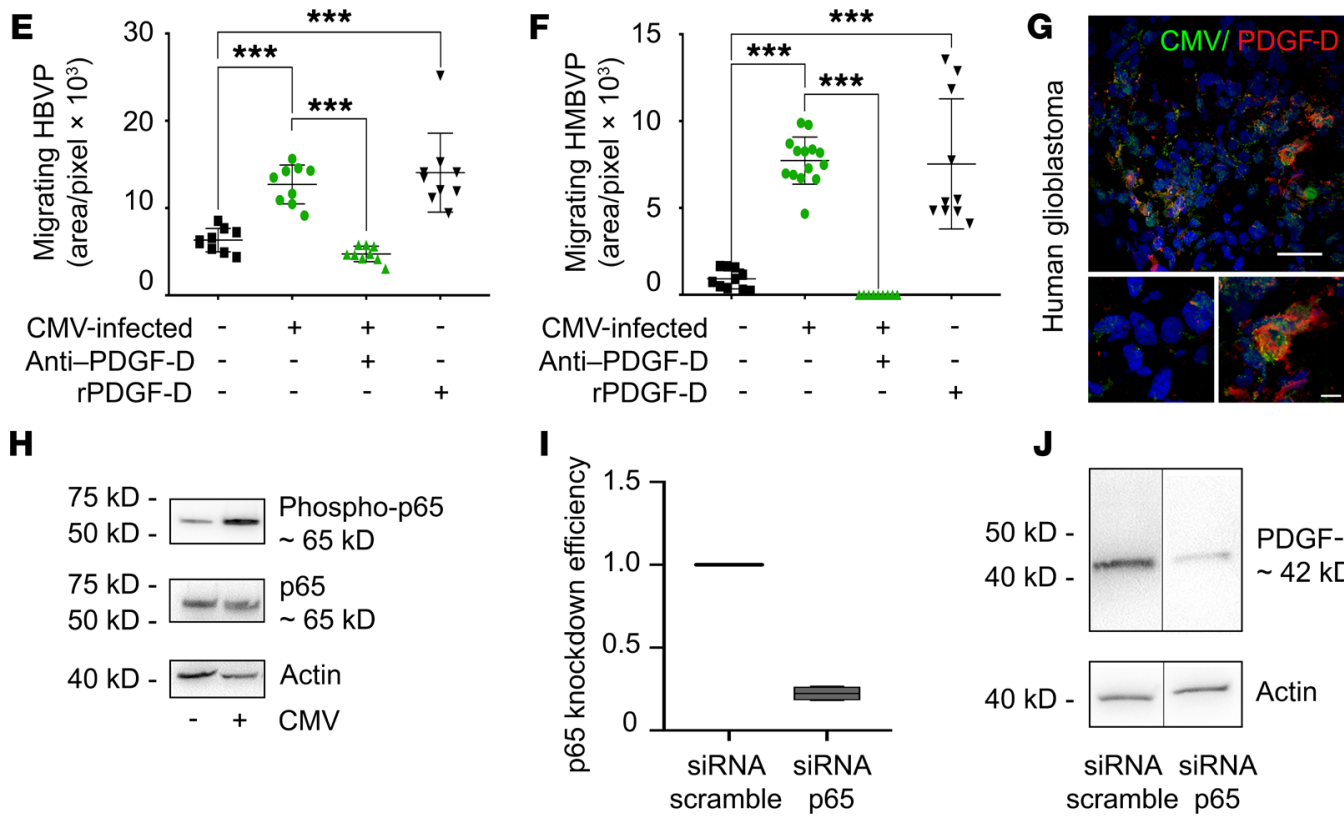

J

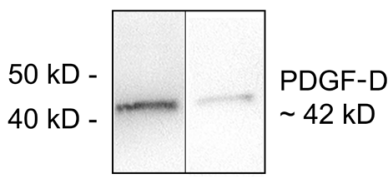

$40 \mathrm{kD}$

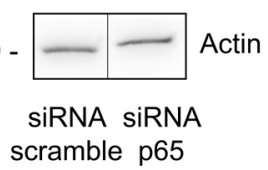

Figure 5. PDGF-D upregulation by CMV in GBM. (A) qRT-PCR for PDGF-D in GSCs after HCMV infection. Box extends from 25th to 75th percentile, and median is indicated by horizontal line. Whiskers represent the maximum and minimum values. Outlier is shown by a green circle. (B) Western blot for PDGF-D in GSCs after HCMV infection. (C) qRT-PCR for PDCF-D in GL261Luc2 cells after MCMV infection. Box extends from 25th to 75th percentile, and median is indicated by horizontal line. Whiskers represent maximum and minimum values. (D) Western blot for PDCF-D in GL261Luc2 cells after MCMV infection. qRT-PCR data are represented as mean \pm SD. ${ }^{*} P<0.05$; ${ }^{* *} P<0.005$, Student's $t$ test (A and $\mathbf{C}$ ). (E and $\mathbf{F}$ ) Transwell migration of HBVP and MBVP in the presence and absence of PDCF-D-neutralizing antibodies, or recombinant PDCF-D (rPDCF-D), as indicated. CMV-infected GBM cells and controls were grown on the bottom of a 12-well plate, as shown. Data are represented as mean \pm SD. ${ }^{* * *} P<0.005$, Holm-Šídák test. (C) PDCF-D (red) and CMV (green) immunofluorescence showing areas of high CMV (lower right) and low CMV (lower left) in human GBM. DAPI-stained nuclei are shown in blue. Scale bar: $50 \mu \mathrm{m}$ (upper images) $10 \mu \mathrm{m}$ (lower images). (H) Increased phosphorylation of NF-KB p65 subunit RelA in G44 GSCs 24 hours after infection with HCMV Towne strain. (I) siRNA-mediated knockdown of RelA in G44 GSCs 48 hours after transfection. Box extends from 25th to 75th percentile, and median is indicated by horizontal line. Whiskers represent maximum and minimum values. (J) PDGF-D downregulation in response to HCMV infection after RelA knockdown in G44 GSCs.

When explanted mouse aortic rings were exposed to media from MCMV-infected GL261Luc2 cells or infected mouse brain vascular pericytes (MBVPs), there was increased endothelial cell sprouting, with the combination of both media showing the greatest effect (Figure $4 \mathrm{C}$ ). Taken together, these data suggest a model in which CMV infection of GBM cells and/or pericytes induces a proangiogenic secretome.

$C M V$-induced $P D G F-D$ regulates pericyte recruitment and angiogenesis in vitro. Consistent with the effects of CMV-conditioned medium on angiogenesis, RNA-Seq of human GSCs after HCMV infection showed angiogenesis as a prominent Gene Ontology (GO) term. To prioritize genes further, we compared upregulated genes after HCMV infection of G44 GSCs with a curated list of secreted proangiogenic proteins. This identified 6 mRNAs with potential proangiogenic roles upregulated in HCMV-infected GSCs: BMP4 (33), CCL2 (34), CXCL8 (35), LIF (36), WNT4 (37), and PDGFD, as shown in Supplemental Table 1. Of these, PDGF-D, a known regulator of pericyte function (38-40), has not been studied in GBM. Because of the pronounced effect on pericytes in our mouse model, we further investigated the potential role of PDGF-D in mediating the observed phenotypes. Independent validation confirmed that PDGF-D was upregulated in GSCs after HCMV infection at both the mRNA and protein levels, and this upregulation persisted over time in human GSCs after HCMV infection (Figure 5, A and B, and Supplemental Figure 4, A and B). $P d g f d$ mRNA and protein upregulation were also observed in MCMV-infected murine GL261Luc2 cells (Figure 5, C and D). Functional studies showed that migration of both human and 
A

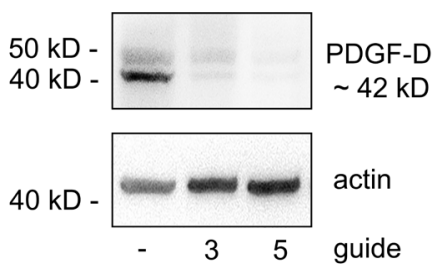

GL261cas9-Pdgfd KO

B 40, GL261cas9-Pdgfd KO
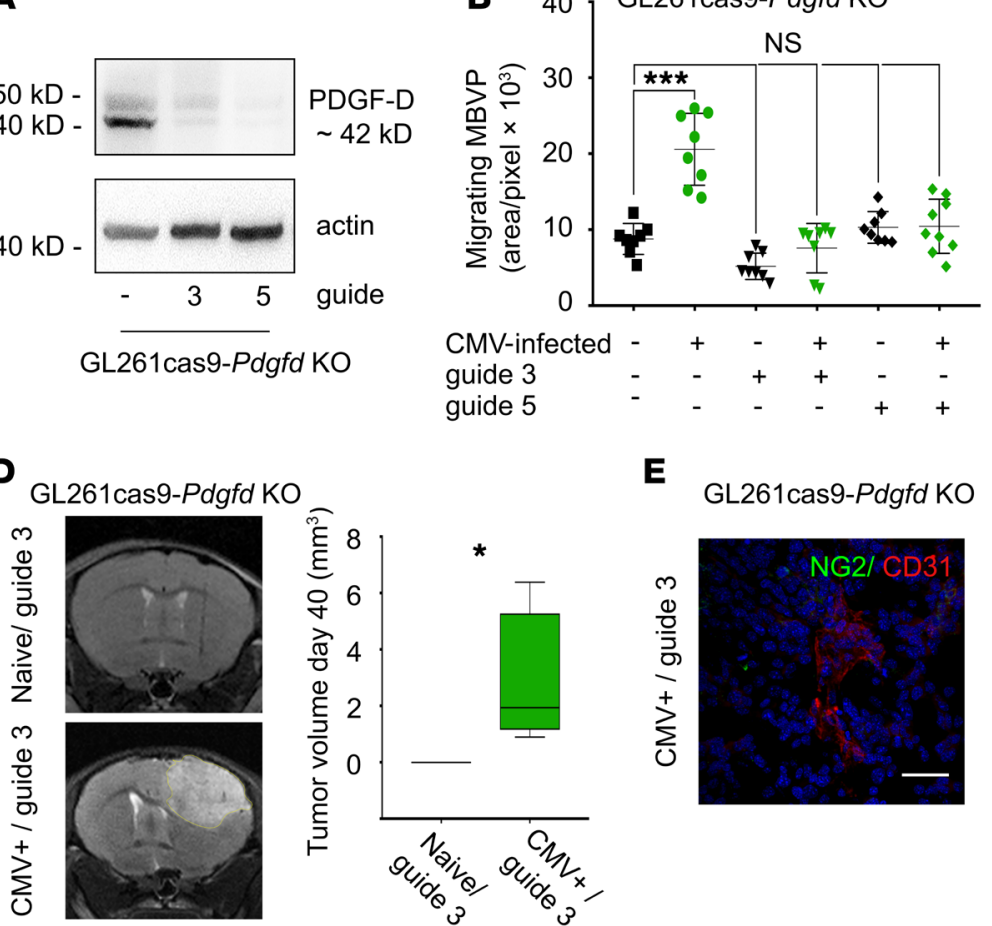

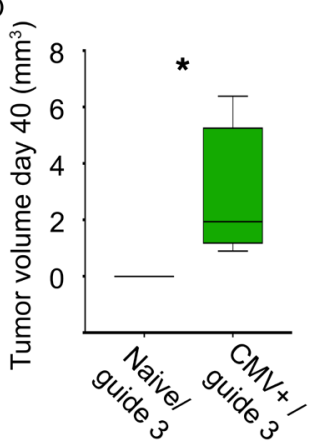

E

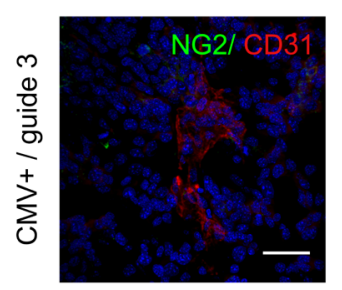

C GL261cas9/

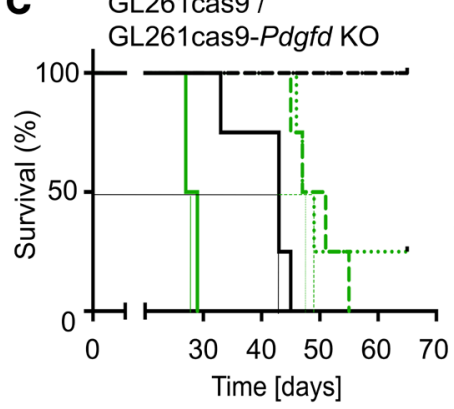

- Naive (43 days)

- - Naive/

guide $3(-)$

.... Naive

guide $5(-)$

$-\mathrm{CMV}+(28$ days $)$

$--\mathrm{CMV}+1$

guide 3 (49 days)

. CMV+ 1

guide 5 (48 days)

$\mathbf{F}$

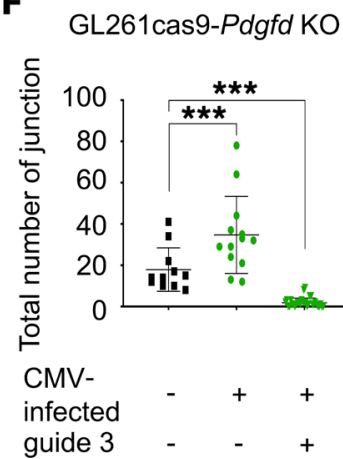

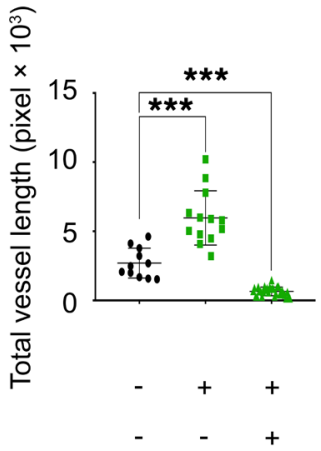

Figure 6. PDGF-D mediates the effects of MCMV in murine GBM. (A) Western blot for PDCF-D in GL261cas9-Pdgfd-KO cells. (B) Transwell migration assay of MBVPs toward GL261cas9-PDGFD-KO cells. Data are represented as mean \pm SD. ${ }^{* * *} P<0.005,2$-way ANOVA. (C) Kaplan-Meier curves of naive and MCMV+ mice intracranially implanted with GL261Cas9 $(n=4)$, GL261cas9-Pdgfd-KO (guide 3) $(n=4)$, or GL261cas9-Pdgfd-KO guide 5 ( $n=4)$. Median survival is indicated on plot and shown in parentheses. (D) T2-weighted MRI at day 30 . Box extends from 25th to 75 th percentile, and median is indicated by horizontal line. Whiskers represent the maximum and minimum values. ${ }^{*} P<0.05$, 2-way ANOVA. (E) CD31 (red), NG2 (green), and nuclei (blue) immunofluorescence in GL261Pdgfd-KO tumors at end points. Scale bar: $50 \mu \mathrm{m}$. (F) Quantitative analysis of angiogenesis and vessel morphology in tumor sections. Data are represented as mean $\pm \mathrm{SD}$. ${ }^{* *} P<0.01 ;{ }^{* *} P<0.005,2$-way ANOVA.

murine pericytes toward CMV-infected GBM cells was abolished by a PDGF-D-neutralizing antibody, while recombinant PDGF-D induced vigorous pericyte migration (Figure 5, E and F). In addition, endothelial tube formation by HCMV-infected GSC-conditioned medium was partially blocked by PDGF-D-neutralizing antibodies, and recombinant human PDGF-D induced tube formation, although to a lesser extent than CMV-infected cell-conditioned medium (Supplemental Figure 4C). Immunofluorescence showed that PDGF-D was widespread in $\mathrm{MCMV}^{+}$ GL261Luc2 tumors and in human GBM specimens, where it partially colocalized with HCMV pp65 staining (Figure 5G and Supplemental Figure 4D). In contrast, intratumoral PDGF-D was barely detectable in MCMV-naive controls (Supplemental Figure $4 \mathrm{D})$, and in normal human brain, PDGF-D staining was confined only to vasculature (Supplemental Figure 4E). Taken together, these data suggest that CMV-induced PDGF-D expression may play an important role in GBM biology.

To further investigate the upregulation of PDGF-D expression after CMV infection, we examined the potential signaling pathways involved. It has been previously reported that CMV infection leads to activation of the NF-кB-signaling pathway (41, 42). It has also been reported in ChIP-Seq studies that PDGF-D expression may be NF- $\mathrm{BB}$ regulated (43); therefore, we investigated this pathway in our GSCs. Western blotting against the phosphorylated p65/RELA subunit of NF- $\mathrm{kB} 24$ hours after infection of G44 GSCs with HCMV indicated that the pathway was activated (Figure 5H). Treatment of G44 GSCs with siRNA against RELA/p65 efficiently knocked down RELA (Figure 5I) and ablated upregulation of PDGF-D in response to HCMV infection, as shown by Western blotting (Figure $5 \mathrm{~J}$ ). Thus, NF- $\mathrm{kB}$ is involved in PDGF-D upregulation by HCMV in GSCs.

Pdgfd knockdown impairs GBM growth in vivo. To further investigate the role of PDGF-D in CMV-induced GBM growth, Pdgfd was knocked out ( $P d g f d$-KO) in GL261 cells using CRISPR/ cas9 technology (Figure 6A). Loss of PDGF-D expression was verified by Western blotting (Figure 6A). CMV infection did not restore PDGF-D expression in Pdgfd-KO cells (Supplemental Figure 5A). Pdgfd-KO cells showed growth kinetics and MCMV infection similar to those of control cells in vitro (Supplemental Figure 5B), but showed impaired pericyte attraction compared with control cells after MCMV infection (Figure 6B). Consistent with our model, orthotopically implanted PDGF-D-competent GL261Cas9 cells grew as tumors, with mortality significantly enhanced in $\mathrm{MCMV}^{+}$mice compared with naive mice (Figure 6C). In contrast, MCMV-naive mice receiving Pdgfd-KO tumor cells all survived more than 60 days (Figure 6C), suggesting a critical role for PDGF-D in GBM progression. Interestingly, lethality was restored when Pdgfd-KO tumors were implanted in $\mathrm{MCMV}^{+}$mice, albeit it was significantly delayed compared with what occurred with PDGF-D-competent tumors (Fig- 

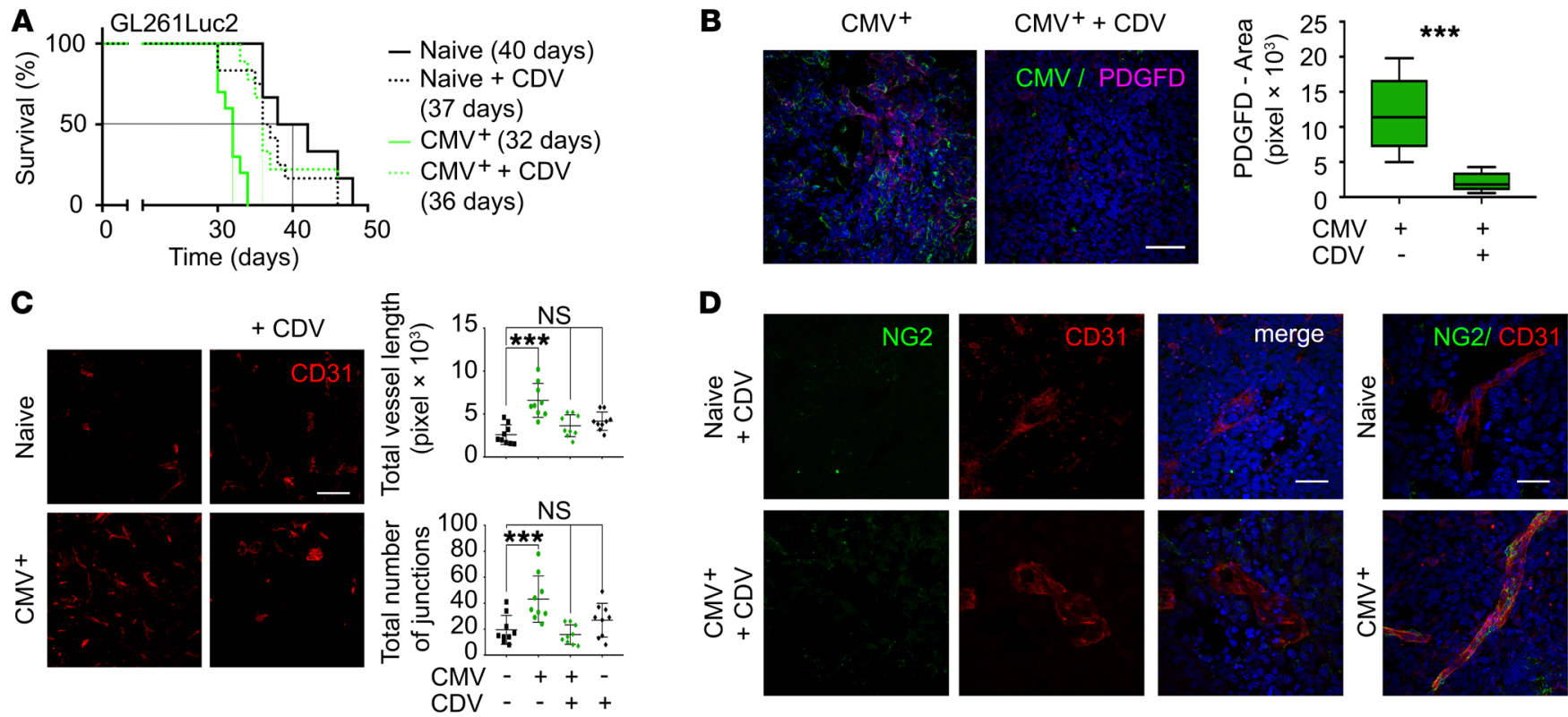

Figure 7. Cidofovir reverses CMV-induced proangiogenic phenotype in MCMV+ GBM mice. (A) Kaplan-Meier survival curve of naive and MCMV+ GL261Luc2 tumor-bearing mice treated with cidofovir (CDV). Median survival is indicated on plot and shown in parentheses. (B) CMV (green, Virusys CA150-1 antibody) and PDCF-D (magenta) immunofluorescence of tumor sections from CDV-treated mice. DAPI-stained nuclei are shown in blue. Scale bar: $100 \mu \mathrm{m}$. Box plot shows quantitation of PDGF-D fluorescence intensity $(n=3)$. Box extends from 25th to 75th percentile, and median is indicated by horizontal line. Whiskers represent maximum and minimum values. ${ }^{* *} P<0.005$, Student's $t$ test. Error bars indicate SD. (C) Quantitative analysis of angiogenesis and vessel morphology after CDV treatment. Scale bar: $100 \mu \mathrm{m} .{ }^{* *} P<0.005$, 2-way ANOVA. Error bars indicate SD. Scale bar: $50 \mu \mathrm{m}$. (D) CD31 (red), NG2 (green), and nuclei (blue) immunofluorescence in tumor sections at survival end points after CDV treatment.

ure 6C). MRI performed at day 40 confirmed Pdgfd-KO tumor establishment only in $\mathrm{MCMV}^{+}$animals (Figure 6D). Pdgfd-KO tumors showed reduced accumulation of pericytes and reduced vessel coverage (Figure 6E), with fewer vessels and branch junctions compared with PDGF-D-competent controls, consistent with impaired angiogenesis (Figure 6F). Together, these results demonstrate an essential role for PDGF-D in GBM growth that can be rescued by MCMV.

The antiviral drug cidofovir reverses the growth-promoting effects of MCMV in the mouse GBM model. The presence of elevated MCMV mRNA and protein after tumor implantation suggested importance of viral activity in our tumor-progression phenotype. To confirm this, $\mathrm{MCMV}^{+}$mice were treated with the viral DNA synthesis inhibitor cidofovir (44) after tumor implantation. Whereas cidofovir treatment had no effect on the survival of naive GL261Luc2 tumor-bearing mice, it significantly improved survival in $\mathrm{MCMV}^{+}$mice compared with untreated controls (Figure 7A). These observations suggest that, under these treatment conditions, cidofovir does not have direct antitumor effects, as tumor growth in MCMV-naive mice was unchanged. Consistent with a direct antiviral effect, MCMV immunostaining was markedly decreased in cidofovir-treated $\mathrm{MCMV}^{+}$mice, and this was associated with dramatically decreased PDGF-D staining and reduction of tumor vascularization to CMV-naive levels (Figure 7, B and C). Finally, cidofovir treatment led to the reversal of MCMV-induced vascular pericyte accumulation (Figure 7D). Together, these results suggest that CMV acts as a critical potentiator of tumor progression by stimulating PDGF-D expression, pericyte accumulation, and GBM angiogenesis.

\section{Discussion}

Here, we provide what we believe is the first report of an in vivo mechanism underlying the promotion of GBM growth by CMV. We have shown that MCMV infection clearly potentiates tumor growth in implantable intracranial murine GBM models. We identified PDGF-D as an essential factor for intracranial GBM growth whose expression is induced by CMV infection of GSCs and stimulates both pericyte attraction and angiogenesis. Importantly, CMV-stimulated tumor growth is reversed by treatment with cidofovir, an FDAapproved antiviral agent (44). Together, these data provide support for the potential clinical importance of CMV in GBM progression and suggest both CMV and PDGF-D as targets in GBM therapy.

Proangiogenic effects of CMV. The most striking effects in $\mathrm{CMV}^{+}$ mice were on angiogenesis. We observed (a) increased intratumoral blood vessel length and branching, (b) increased pericyte coverage of intratumoral blood vessels, (c) increased intratumoral blood flow, (d) a proangiogenic secretome in CMV-infected pericytes, and (e) a proangiogenic and pericyte-attracting secretome in CMV-infected GBM cells. These observations support the concept that a CMV-mediated increase in vessel number and maturity is likely to be a major factor in the increased tumor growth in the context of the $\mathrm{MCMV}^{+}$mice. Indeed, it has been established that CMV has proangiogenic effects in nontumoral settings (45-47), and a role in tumor angiogenesis has been previously speculated, although never established (48). One of our most striking observations was the striking increase in pericyte coverage of the tumor vasculature in $\mathrm{MCMV}^{+}$animals. Pericytes are permissive for CMV infection (28), and indeed, these tumor-associated pericytes strongly stain for MCMV in both our model and in human tumors. 
Pericytes are essential for fully functional brain vasculature (49), and a direct correlation between pericyte coverage of tumor vasculature and diminished survival in patients with GBM has been reported, highlighting the relevance of this observation (31). Overall, we examined tumor samples from 5 patients using immunofluorescence and 18 using RT-PCR (Figure 3 and Supplemental Figure 3). Of those, all 5 immunostained samples were positive for HCMV pp65 and only 1 sample from the PCR study was negative for CMV. Although CMV reactivity was predominantly found in the perivascular niche, single cells remained positive throughout the tumor. Further studies on an extended panel of tumors are warranted.

$C M V$ reactivation in the mouse model. As reported in human specimens, MCMV reactivation could be detected in our mouse model. We observed (a) a time-dependent increase of MCMV IE1/m123 levels as tumors grew and (b) the presence of CMV in intratumoral pericytes as well as tumor cells by immunostaining in both the mouse model and in human GBM specimens.

This is the first report, to our knowledge, to show that CMV is reactivated in perivascular intratumoral pericytes, suggesting potentially important biological mechanisms. We speculate that CMV reactivation occurs within the tumor microenvironment due to local immunosuppression that prevents control of CMV reactivation by the adaptive immune system. Based on our observations, we also speculate that at least one source of intratumoral CMV may be pericytes recruited from the circulation and are currently investigating this hypothesis. Indeed, pericytes are known to be permissive for CMV infection and may play a role in CMV retinitis (28). Our data so far suggest that CMV reactivation is specific to the immunosuppressive tumor microenvironment. We were unable to see any symptoms of systemic CMV reactivation or detect its presence in lung tissue from MCMV-infected tumor-bearing animals. However, this does not discount the possibility that CMV may be reactivated in other tissues or specific cell types during tumor growth. Also, there are many additional CMV-encoded transcripts we could search for. This is an area of current investigation.

One of the challenges in this field of study is to relate changes seen in vitro under acute infection conditions, to those seen in vivo when the virus is undergoing long term reactivation in multiple cell types within the tumor microenvironment. Our mouse model will allow highly detailed studies of viral gene reactivation and other molecular changes temporally and by cell type enabling a detailed understanding of the dynamics of the process. Measurement of MCMV transcript levels showed a 10-fold lower expression of IE1/m123 in vivo compared with in vitro by qRT-PCR. On a cellular level, this difference is likely smaller, due to the lower numbers of cells infected in vivo versus in vitro. Initial comparisons of CMV IE1/m123 levels between the murine model and human GBM specimens suggest that levels of CMV activation may be slightly higher in the murine model (data not shown). However, this does not account for the variable of numbers of cells infected per specimen and changes in CMV activation over tumor evolution. Further detailed studies are therefore needed to survey multiple genes and specimens in each system.

PDGF-D as a mediator of the effects of CMV on pericyte recruitment and angiogenesis. In our system, CMV infection of GSCs promotes the secretion of a wide array of different proangiogenic cytokines, a phenotype previously observed in other cell types (45-48). Com- parison of our RNA-Seq data with GO terms for secreted angiogenic molecules revealed several upregulated mRNAs encoding secreted proteins involved in the promotion of angiogenesis in GBM by CMV, as shown in Supplemental Table 1, suggesting that promotion of angiogenesis is multifactorial. Interestingly, our RNA-Seq data scored highly for PDGF-D, which is known to be involved in the control of pericyte migration (50), but has not been studied in the context of GBM. Similarly to PDGF-B, PDGF-D is a ligand of PDGFR- $\beta$, and null mouse mutants of either PDGFR- $\beta$ or PDGF-B are lethal in utero due to hemorrhage caused by abnormal, pericyte-lacking vasculature (51). Additionally, PDGF-D is known to be involved in pericyte, macrophage, and monocyte chemotaxis as well as the induction of angiogenesis in vitro $(52,53)$.

Our in vitro data suggest that PDGF-D is responsible for pericyte accumulation in $\mathrm{GBM}$ in $\mathrm{CMV}^{+}$mice and also contributes to angiogenesis. We showed by qRT-PCR and Western blotting that PDGF-D is clearly upregulated in human GSCs and mouse GBM cells after infection with CMV, and we confirmed that PDGF-D alone can stimulate pericyte migration in both mouse and human systems. Depletion of PDGF-D by blocking antibodies or CRISPR-mediated gene editing completely blocks pericyte migration in vitro. Most compelling is the observation that GBM cells lacking PDGF-D cannot grow in CMV-naive hosts, showing lack of both pericyte influx and angiogenesis. However, when these same KO cells are introduced into $\mathrm{MCMV}^{+}$mice, tumor growth and lethality are restored, although more slowly than in WT controls, suggesting that extratumoral factors provided by MCMV in the model may overcome the lack of PDGF-D in tumor cells. Whether CMV is providing PDGF-D or a different compensatory factor is a subject of current investigation. Our data suggest the importance of PDGF-D as a potential therapeutic target in vivo.

Regulation of PDGF-D expression by $N F-\kappa B$. We showed that PDGF-D upregulation is at least in part regulated by CMV activation of NF- $\kappa$ B signaling (Figure $5, \mathrm{H}-\mathrm{J}$ ). It has been previously shown that, after HCMV infection, cells show a virus-regulated induction of NF- $\kappa B$ signaling (41). Purified viral glycoproteins were shown to induce NF- $\kappa \mathrm{B}$ activity $(41,42)$. In this study, we showed that in human GSCs, infection with CMV results in the activation of NF- $\kappa \mathrm{B}$ signaling via $\mathrm{p} 65$ phosphorylation. Because NF- $\kappa \mathrm{B}$ has also been associated with PDGF-D in ChIP-Seq studies (43), we hypothesized that NF- $\kappa$ B activation might also lead to PDGF-D upregulation. This was confirmed by siRNA-mediated inhibition of NF- $\kappa \mathrm{B}$ signaling. As the induction of PDGF-D expression by CMV was only partially inhibited, other mechanisms, such as Sp-1 induction upon CMV infection, might also be involved in PDGF-D upregulation $(54,55)$.

Effects of antiviral therapy in CMV GBM models. In recent years, clinical approaches targeting CMV have led to encouraging clinical results (13-18). These approaches include CMV-targeted immunotherapies and the antiviral drug valganciclovir, which is a substrate of CMV thymidine kinase (TK), rendering infected cells exquisitely sensitive to its effects by accumulation of toxic metabolites catalyzed by viral TK activity. However, MCMV does not have a homolog of the HCMV TK gene and is not sensitive to valganciclovir. Therefore, in the current report, we tested the effects of cidofovir, an FDA-approved antiviral drug that inhibits viral DNA polymerase and blocks MCMV 
replication. Treatment of $\mathrm{MCMV}^{+}$GL261 tumor-bearing mice with cidofovir caused reversal of the MCMV-induced phenotype, with a reduction of tumor-infiltrating pericytes, decreased tumor vasculature, and improved survival, consistent with these clinical observations. Previous work has suggested that cidofovir has antitumor potential both in vitro and in vivo in combination with irradiation (56). The use of cidofovir as an anticancer drug is not restricted to GBM and has been shown to be effective in HPV-associated cervical cancer (57), although with some toxicity (58). Thus, the current report suggests that cidofovir might improve survival in hosts with CMV, but it is not clear that CMV-naive hosts would show such a benefit. Our observation that cidofovir treatment could reverse the effects of $\mathrm{CMV}$ on GBM growth provides at least proof of principle supporting the potential use of antiviral treatments in brain tumors. Importantly, others have shown potentially favorable data on the use of valganciclovir in patients (19) and have also shown that cidofovir can sensitize cells to irradiation (56). This suggests that incorporating irradiation into our model would show further enhancement of animal survival and would strongly support clinical application. Studies are currently underway with additional antiviral drugs in combination with other therapies to assess the translational relevance of this approach.

Limitations. This study was performed in mouse GBM models, and MCMV has many differences from HCMV. The lack of TK in MCMV limits our ability to test valganciclovir in this model; however, an engineered MCMV with a copy of HCMV TK has been created (59), and we will investigate this in order to model the effects of valganciclovir. CMV and its relationship with GBM and cancer remains a controversial area, with some groups unable to detect CMV in human specimens $(22,23)$. At present, there are no reports of next-generation sequencing data from human tumor specimens containing CMV sequences, which may be explainable by low levels of CMV in tumors as well as a high level of natural genetic variation in CMV. Also, until this study, there was no mechanistic explanation, to our knowledge, of how CMV could influence tumor growth. Our observations therefore add to the weight of evidence that CMV can play a role in tumor growth and may be therapeutically very important.

Summary and perspective. Our observations provide insights into how CMV infection affects GBM growth in vivo and are supported by observations in human patient specimens. These data strengthen the argument for a role of CMV in GBM growth and a rationale for antiviral therapy in overcoming treatment limitations in current GBM therapy. Finally, in identifying PDGF-D as a contributor to the CMV-induced phenotype, we have identified a target for potentiating current GBM therapy regimens, augmenting immunotherapeutic approaches, and ultimately, developing new approaches to treating GBM.

\section{Methods}

Cell culture and virus propagation. NIH3T3 mouse fibroblasts and MRC- 5 cells were purchased from ATCC and cultured in DMEM with 10\% FBS (Sigma-Aldrich), penicillin (100 U/ml), and streptomycin $(10 \mathrm{mg} / \mathrm{ml}$ ) (Thermo Fisher). MCMV lacking the m157 gene (24) was provided by Ulrich Koszinowski (Ludwig-Maximilians-Universitat, Munich, Germany), and HCMV-GFP Towne strain was pro- vided by Bill Britt (University of Alabama, Birmingham, Alabama, USA). MCMV and HCMV strains were cultured in NIH3T3 and MRC- 5 cells, respectively, and viral titer was measured as previously described (24). GL261Luc2 murine glioma cells were purchased from PerkinElmer. CT-2A murine glioma cells were a gift from Thomas Seyfried (Boston College, Boston, Massachusetts, USA). HBMEC cells were purchased from ScienCell Research Laboratories and grown according to the manufacturer's recommendations. Primary human GSCs (G34, G35, G44, G157) were obtained by dissociation of gross tumor samples and cultivated in neurosphere media, as previously described (60). Mycoplasma testing was routinely done by PCR. GBM subtype classification was done by gene-expression profiling as previously described (60).

$C M V$ infection in vitro. For CMV infection in vitro, we seeded up to $10^{6}$ cells in 6-well plates and treated with CMV or mock infection (purified extract from uninfected fibroblasts) the next day. We infected GL261Luc2 neurospheres with MCMV (MOI of 1) or patient-derived human GSCs with HCMV (MOI of 1) for 2 hours. Cells were then rinsed with Dulbecco's PBS (DPBS) (Thermo Fisher) and covered with fresh culture medium.

Transwell cell migration assay. Cells were seeded in a 24-well culture plate, infected with CMV (MOI of 1), and cultured for 72 hours. Light transmission blocking Transwell inserts (FluoroBlock, Corning) with a pore size of $8 \mu \mathrm{m}$ were used. $1 \times 10^{6}$ Cells were trypsinized and stained with Vybrant DiO Cell-Labeling Solution (Thermo Fisher). Cells were added to the Transwell compartment and incubated at $37^{\circ} \mathrm{C}$. Images were taken after 24 hours and 48 hours. For each assay, 15 images from 3 separate wells were analyzed. Each assay was repeated 3 times.

Flow cytometry. Flow cytometry was performed on a FACS LSR II or Fortessa (BD Biosciences). Cells were gated by forward scatter/ side scatter while excluding duplets by forward scatter area/forward scatter height. Subsequently, CD $45^{+}$(BioLegend, catalog 103101) cells were gated, with further classification by their expression of NG2 (Stratech, catalog bs-4800R-FITC) and PDGFR- $\beta$ (BioLegend, catalog 136007).

qRT-PCR. Total RNA was extracted using TRIzol and treated with RNase-free DNase (QIAGEN). mRNA expression analysis was carried out using Power SYBR Green (Applied Biosystems). RNA concentration was quantified using a Nanodrop RNA 6000 (Thermo Fisher) and analyzed using the Applied Biosystems StepOnePlus PCR machine (Thermo Fisher). See Supplemental Table 2 for primer sequences.

Generation of knockdown cell lines using siRNA. Human RELA was knocked down using siRNA technology. Briefly, GSCs were transfected using siRNA oligonucleotides against p65/RELA (QIAGEN) or nontargeting controls at $100 \mathrm{pmol} / 5 \times 10^{5}$ cells and validated at the mRNA and protein levels. Cells were processed 48 hours after transfection. All transfections were performed with Lipofectamine 2000 (Life Technologies) following the manufacturer's recommendations. Antibodies used were as follows: NF- $\kappa \mathrm{B}$ p $65 \mathrm{XP}$ rabbit $\mathrm{mAb}$ (clone D14E12, catalog 8242), phospho-NF-кB p65 (Ser536) mouse mAb (clone E1Z1T, catalog 13346) (Cell Signaling Technology).

In vivo studies. Six-week-old male and female C57BL/6 mice were purchased from The Jackson Laboratory and mated once. The f1 generation was inoculated with a nonlethal dose of $\triangle 157$ MCMV Smith strain at P2 (10 3 PFU) by i.p. injection, as previously described (24). After 14 weeks, 1000 cells (GL261Luc2, GL261Cas9, GL261PDGFKO, 
and CT-2A) in $3 \mu \mathrm{l}$ normal saline were injected intracranially to establish mouse brain tumors ( $2 \mathrm{~mm}$ right lateral, $1 \mathrm{~mm}$ frontal to the bregma, and $3 \mathrm{~mm}$ deep). Infection was confirmed in serum samples using the commercial pathology service provided by Charles River (anti-MCMV Multiplexed Fluorometric ImmunoAssay). Cidofovir treatment was performed by i.p. injection $(100 \mathrm{mg} / \mathrm{kg}) 3$ times per week for 2 weeks. DMSO and sterile saline were used as vehicle.

Imaging methods. MRI data were acquired using a Bruker 7 Tesla scanner (Bruker Biospin). Animals were kept under isoflurane narcosis throughout the scan. Respiration and heart rate were monitored. Body temperature was maintained using a homeothermic blanket. ASL was performed as previously described (61). Briefly, the animal was carefully positioned with the labeling coil located at the neck to allow labeling of blood flowing through the carotid arteries. For ASL, single-shot, gradient-echo, echo-planar imaging (EPI) acquisition was used. Paired images were acquired alternately - one with ASL (labeled image) and the other without (control). Tumor blood flow was analyzed by comparing specified regions of interest (ROIs) with their anatomical parallel in the contralateral hemisphere. The average difference in ROIs was compared between different animals and translated into relative fold-change difference. T2-weighted images were acquired using the RARE pulse sequence. The resulting segmentations were reviewed manually to ensure accuracy.

Generation of KO cell lines using CRISPR/Cas9. The murine Pdgfd gene was knocked out using CRISPR/Cas9 technology. Briefly, GL261 cells were lentivirally transduced with the Cas9 gene. Stable Cas9expressing clones were then transduced with a lentivirus expressing a gRNA specifically designed to target the murine Pdgfd gene (designated as guide 3 and guide 5). The plasmid constructs used to make the lentivirus vectors, pLentiCAS9Blast and pLentiGuide (guide 3 targets exon 2 of the gene: $5^{\prime}$-GGGTAGCTGTTCGGGAAGCG-3'; guide 5 targets exon 3 of the gene: $5^{\prime}$-TTTGTTCTTGACGTTATCCT- 3 ') were purchased from GenScript. Cells successfully transfected with the gRNA were positively selected using puromycin resistance, and single clones were isolated via serial dilution. Only clones testing negative for PDGF-D expression by Western blot analysis were used.

RNA-Seq. Triplicates of proneural GSCs (G44) were infected with HCMV at an MOI of 0.1. Cells were then kept in culture for 3 days. Uninfected cells served as controls. Equal quantities of total RNA were isolated using the TRIzol reagent (Thermo Fisher) according to the manufacturer's protocol. RNA degradation and contamination were monitored on $1 \%$ agarose gels, and RNA integrity was assessed with the RNA Nano 6000 Assay Kit and a Bioanalyzer 2100 system (Agilent Technologies). Sequencing libraries were generated using the Illumina paired-end indexing protocol. Corresponding RNASeq paired-end reads were processed using the TopHat suite with Cufflinks. Raw reads in fastq format were mapped to the reference organism using STAR software. Clean data were obtained by removing reads containing adapter sequences, reads containing poly-N, and low-quality reads from raw data. The Q20, Q30, GC-content, and sequence duplication levels of the clean data were calculated. All downstream analyses were based on high-quality clean data. All RNAseq data were deposited in the EMBL-EBI's ArrayExpress database (E-MTAB-7613).

Analysis was performed using the Kyoto Encyclopedia of Genes and Genomes (KEGG) for pathway enrichment analyses and Protein ANalysis THrough Evolutionary Relationships (PANTHER) for GO.
Immunoblotting. Cells were lysed using RIPA buffer containing 1\% protease inhibitor cocktail (Merck Millipore) and 5\% phosphatase inhibitor cocktail (Roche). Total protein concentration was measured using the Bradford protein assay. Primary antibodies used were against HCMV (Virusys Corporation, catalog CA150-1; 1:1000), HCMV pp65 (Virusys, catalog CA003-100; 1:1000), anti-human PDGF-D (Thermo Fisher, catalog 40-2100; 1:1000), and $\beta$-actin (Cell Signaling Technology, catalog 4967; 1:1000).

Immunohistochemistry. Mice were euthanized using $\mathrm{CO}_{2}$ inhalation and subsequently perfused with $4 \%$ neutral-buffered formalin (Sigma-Aldrich) for fixation. Cryoprotection was performed using $30 \%$ sucrose. All mouse brain slides were obtained from $30 \mu \mathrm{m}$ frozen sections. Permeabilization was done using 1\% Triton X-100 (SigmaAldrich) in PBS (Thermo Fisher) for 10 minutes. Slides were then incubated with the primary antibody (1:100 in normal serum) overnight at $4^{\circ} \mathrm{C}$. For detection of the primary antibody, species-matched fluorophore-coupled antibodies were incubated for 1 hour at room temperature. Slides were then covered with antifade mounting medium (Vectashield, Vector Laboratories) and coverslipped. All fluorescent and bright-field microscopy-based assays were observed using a Nikon Eclipse Ti microscope (Nikon). High-resolution confocal fluorescent microscopy was performed using a Zeiss LSM 710 confocal microscope system and visualized using ZEN Zeiss Imaging software. For human specimens, IHC staining was performed as previously described (6). Briefly, tissue specimens were incubated with antibodies against CMV (Virusys Corp.), followed by H\&E counterstaining. We first validated the MCMV immunostaining conditions in vitro by staining of MCMV-infected and noninfected GL261 cells. Additional controls used for antibody specificity were brain and tumors from noninfected control animals, brain subventricular zone staining in infected animals, and the absence of any background staining with secondary antibodies alone. For colocalization, images were imported into the Fiji version of the free image-processing software ImageJ (NIH). The preinstalled plugin for colocalization analysis coloc2, which uses a pixel intensity correlation measurement, was used to calculate colocalization parameters (Pearson's coefficient and Spearman's rank correlation). For immunofluorescence studies, the following antibodies were used: antimouse, CD31 (Bio-Rad, catalog TLD-3A12, 1:100), PDGF-D (Thermo Fisher, catalog 40-2100, 1:100), and PDGFR $\beta$ (BioLegend, 323605, 1:100); anti-human, PDGF-D (R\&D, catalog AF1159, 1:100), CD133 (BioLegend, catalog S16016B, 1:100), Vimentin (SP20, Thermo Fisher, catalog MA5-16409, 1:100), and CD31 (Bio-Rad, catalog MCA1738, 1:100); anti-human mouse Ki67 (Abcam, catalog ab15580, 1:100), NG2 (EMD Millipore, 1:100), and Olig2 (Merck, catalog MABN50, 1:100); anti-CMV (Virusys Corp., catalog CA150-1, 1:1000) and HCMV pp65 (Virusys, catalog CA003-100, 1:1000).

Quantification of tumor vasculature in frozen sections. Immunofluorescence staining using anti-CD31 antibodies (Bio-Rad, catalog MCA2388GA) was performed in coronal frozen brain sections. An Image J algorithm (provided by Institute for the Neurosciences, Neuro Technology Studio, Brigham and Women's Hospital) was used to automate vessel detection and characterization for length, area, and branching points (25).

Statistics. All microscope-based assays were edited/quantified using ImageJ, including the Analyze Particles function of binary images with automatic threshold. Data are expressed as mean \pm SD. Unpaired 2-tailed Student's $t$ test was used for comparison between 
2 groups. Each group was tested for Gaussian distribution, if 1-way ANOVA was passed, followed by Bonferroni's test. If this failed, the Kruskal-Wallis test followed by Dunn's correction was conducted to test for significance among multiple groups. Pearson's correlation with nonlinear regression analysis was performed to compute Pearson's $r$, R2, and $P$ values. The Holm-Šídák test was used for some experiments, as indicated in the figure legends. Statistical analyses were performed using Microsoft Office Excel 2011 or Graph Pad Prism 6 software. $P<$ 0.05 was considered statistically significant.

Study approval. Tumor samples were obtained using a protocol approved by the Dana-Farber Cancer Institute IRB. Written, informed consent was received from all participants prior to inclusion in the study. Animal studies were approved by the Brigham and Women's Hospital Center for Comparative Medicine IACUC.

\section{Author contributions}

HK designed the study, acquired and analyzed data, and participated in manuscript writing. $\mathrm{PB}, \mathrm{VL}, \mathrm{CP}, \mathrm{MZ}, \mathrm{MON}, \mathrm{KG}, \mathrm{HZ}, \mathrm{MS}$, HI, MG, MBG, FR, LD, RZ, AR, and SP assisted in experimental design, execution, and data analysis. CDJ and CSC assisted in experimental design and interpretation of data. CHC provided reagents. $\mathrm{CHC}$ and $\mathrm{EAC}$ assisted in experimental design and interpretation and participated in manuscript writing. SEL designed the study, analyzed data, and wrote the paper. All authors discussed the results and commented on the manuscript.

\section{Acknowledgments}

This work was funded in part by the following: NIH grants NCI RO1 CA195532 (to EAC) and NCI R01 CA184283 (to CSC) and an American Brain Tumor Association postdoctoral research fellowship (to $\mathrm{CP}$ ). We thank James Zoldak for critical reading of the manuscript.

Address correspondence to: Sean E. Lawler, Harvey Cushing Neurooncology Laboratories, Department of Neurosurgery, Brigham and Women's Hospital, Hale Building for Transformative Medicine, 60 Fenwood Road, Boston, Massachusetts 02115, USA. Phone: 1.617.525.5650; Email: slawler@bwh.harvard.edu.

HK's present address is: Department of Neurosurgery, University Medical Center Mainz, Germany.
1. Alexander BM, Cloughesy TF. Adult glioblastoma. J Clin Oncol. 2017;35(21):2402-2409.

2. Louis DN, et al. The 2016 World Health Organization Classification of Tumors of the Central Nervous System: a summary. Acta Neuropathol. 2016;131(6):803-820.

3. Stupp R, et al. Radiotherapy plus concomitant and adjuvant temozolomide for glioblastoma. NEngl J Med. 2005;352(10):987-996.

4. Stupp R, et al. Effects of radiotherapy with concomitant and adjuvant temozolomide versus radiotherapy alone on survival in glioblastoma in a randomised phase III study: 5-year analysis of the EORTC-NCIC trial. Lancet Oncol. 2009;10(5):459-466.

5. Mocarski E, et al. Cytomegaloviruses. In: Knipe DM, Howley PM, eds. Fields Virology. 6th ed. Philadelphia, Pennsylvania, USA: Wolters, Kluwer, Lippincott, Williams \& Wilkins; 2013:1960-2014.

6. Cobbs CS, et al. Human cytomegalovirus infection and expression in human malignant glioma. Cancer Res. 2002;62(12):3347-3350.

7. Dziurzynski K, et al. Glioma-associated cytomegalovirus mediates subversion of the monocyte lineage to a tumor propagating phenotype. Clin Cancer Res. 2011;17(14):4642-4649.

8. Rahbar A, et al. Human cytomegalovirus infection levels in glioblastoma multiforme are of prognostic value for survival. J Clin Virol. 2013;57(1):36-42.

9. Bhattacharjee B, Renzette N, Kowalik TF. Genetic analysis of cytomegalovirus in malignant gliomas. J Virol. 2012;86(12):6815-6824.

10. Lucas KG, Bao L, Bruggeman R, Dunham K, Specht C. The detection of CMV pp65 and IE1 in glioblastoma multiforme. J Neurooncol. 2011;103(2):231-238.

11. Harkins LE, et al. Detection of human cytomegalovirus in normal and neoplastic breast epithelium. Herpesviridae. 2010;1(1):8.

12. Chen HP, et al. Human cytomegalovirus preferentially infects the neoplastic epithelium of colorectal cancer: a quantitative and histological analysis. J Clin Virol. 2012;54(3):240-244.

13. Nair SK, Sampson JH, Mitchell DA. Immunological targeting of cytomegalovirus for glioblastoma therapy. Oncoimmunology. 2014;3:e29289.

14. Prins RM, Cloughesy TF, Liau LM. Cytomegalovirus immunity after vaccination with autologous glioblastoma lysate. $\mathrm{N} \mathrm{Engl} \mathrm{J} \mathrm{Med.}$ 2008;359(5):539-541.

15. Mitchell DA, et al. Tetanus toxoid and CCL3 improve dendritic cell vaccines in mice and glioblastoma patients. Nature. 2015;519(7543):366-369.

16. Batich KA, et al. Long-term survival in glioblastoma with cytomegalovirus pp65-targeted vaccination. Clin Cancer Res. 2017;23(8):1898-1909.

17. Reap EA, et al. Dendritic cells enhance polyfunctionality of adoptively transferred $\mathrm{T}$ cells that target cytomegalovirus in glioblastoma. Cancer Res. 2018;78(1):256-264.

18. Schuessler A, et al. Autologous T-cell therapy for cytomegalovirus as a consolidative treatment for recurrent glioblastoma. Cancer Res. 2014;74(13):3466-3476.

19. Stragliotto $G$, et al. Effects of valganciclovir as an add-on therapy in patients with cytomegalovirus-positive glioblastoma: a randomized, double-blind, hypothesis-generating study. Int J Cancer. 2013;133(5):1204-1213.

20. MacManiman JD, et al. Human cytomegalovirusencoded pUL7 is a novel CEACAM1-like molecule responsible for promotion of angiogenesis. MBio. 2014;5(6):e02035.

21. Soroceanu L, et al. Cytomegalovirus immediate-early proteins promote stemness properties in glioblastoma. Cancer Res. 2015;75(15):3065-3076.

22. Lawler SE. Cytomegalovirus and glioblastoma; controversies and opportunities. J Neurooncol. 2015;123(3):465-471.

23. Holdhoff M, et al. Absence of cytomegalovirus in glioblastoma and other high-grade gliomas by real-time PCR, immunohistochemistry, and in situ hybridization. Clin Cancer Res.
2017;23(12):3150-3157.

24. Price RL, et al. Cytomegalovirus contributes to glioblastoma in the context of tumor suppressor mutations. Cancer Res. 2013;73(11):3441-3450.

25. Zudaire E, Gambardella L, Kurcz C, Vermeren S. A computational tool for quantitative analysis of vascular networks. PLOS ONE. 2011;6(11):e27385.

26. Garg AD, et al. Dendritic cell vaccines based on immunogenic cell death elicit danger signals and T cell-driven rejection of high-grade glioma. Sci Transl Med. 2016;8(328):328ra27.

27. Shanley JD, Jordan MC, Cook ML, Stevens JG. Pathogenesis of reactivated latent murine cytomegalovirus infection. Am J Pathol. 1979;95(1):67-80.

28. Alcendor DJ, Charest AM, Zhu WQ, Vigil HE, Knobel SM. Infection and upregulation of proinflammatory cytokines in human brain vascular pericytes by human cytomegalovirus. J Neuroinflammation. 2012;9:95.

29. Nakano M, et al. NG2 glial cells regulate neuroimmunological responses to maintain neuronal function and survival. Sci Rep. 2017;7:42041.

30. Hall CN, et al. Capillary pericytes regulate cerebral blood flow in health and disease. Nature. 2014;508(7494):55-60.

31. Caspani EM, Crossley PH, Redondo-Garcia C, Martinez S. Glioblastoma: a pathogenic crosstalk between tumor cells and pericytes. PLOS ONE. 2014;9(7):e101402.

32. Zhou W, et al. Targeting glioma stem cell-derived pericytes disrupts the blood-tumor barrier and improves chemotherapeutic efficacy. Cell Stem Cell. 2017;21(5):591-603.e4.

33. Piccirillo SG, et al. Bone morphogenetic proteins inhibit the tumorigenic potential of human brain tumour-initiating cells. Nature. 2006;444(7120):761-765

34. Chang AL, et al. CCL2 produced by the glioma microenvironment is essential for the recruitment of regulatory T cells and myeloid-derived suppres- 
sor cells. Cancer Res. 2016;76(19):5671-5682.

35. Brat DJ, Bellail AC, Van Meir EG. The role of interleukin-8 and its receptors in gliomagenesis and tumoral angiogenesis. Neuro-oncology. 2005;7(2):122-133.

36. Peñuelas $S$, et al. TGF-beta increases gliomainitiating cell self-renewal through the induction of LIF in human glioblastoma. Cancer Cell. 2009;15(4):315-327.

37. Daneman R, Agalliu D, Zhou L, Kuhnert F, Kuo CJ, Barres BA. Wnt/beta-catenin signaling is required for CNS, but not non-CNS, angiogenesis. Proc Natl Acad Sci US A. 2009;106(2):641-646.

38. Kumar A, et al. Platelet-derived growth factor-DD targeting arrests pathological angiogenesis by modulating glycogen synthase kinase-3beta phosphorylation. J Biol Chem . 2010;285(20):15500-15510.

39. Gladh H, et al. Mice lacking platelet-derived growth factor D display a mild vascular phenotype. PLOS ONE. 2016;11(3):e0152276.

40. Furuhashi M, et al. Platelet-derived growth factor production by B16 melanoma cells leads to increased pericyte abundance in tumors and an associated increase in tumor growth rate. Cancer Res. 2004;64(8):2725-2733.

41. Yurochko AD, Kowalik TF, Huong SM, Huang ES. Human cytomegalovirus upregulates NF-kappa B activity by transactivating the NF-kappa B p105/p50 and p65 promoters. J Virol. 1995;69(9):5391-5400.

42. Yurochko AD, Hwang ES, Rasmussen L, Keay S, Pereira L, Huang ES. The human cytomegalovirus UL55 (gB) and UL75 (gH) glycoprotein ligands initiate the rapid activation of Sp1 and NF-kappaB during infection. J Virol. 1997;71(7):5051-5059.

43. Satoh J. Molecular network of ChIP-Seq-based $\mathrm{NF}-\kappa \mathrm{B}$ p 65 target genes involves diverse immune functions relevant to the immunopathogenesis of multiple sclerosis. Mult Scler Relat Disord. 2014;3(1):94-106.

44. Chakrabarti S, Collingham KE, Osman H, Fegan CD, Milligan DW. Cidofovir as primary pre-emptive therapy for post-transplant cytomegalovirus infections. Bone Marrow Transplant. 2001;28(9):879-881.

45. Botto S, et al. IL-6 in human cytomegalovirus secretome promotes angiogenesis and survival of endothelial cells through the stimulation of survivin. Blood. 2011;117(1):352-361.

46. Dumortier J, et al. Human cytomegalovirus secretome contains factors that induce angiogenesis and wound healing. JVirol. 2008;82(13):6524-6535.

47. Streblow DN, Dumortier J, Moses AV, Orloff SL, Nelson JA. Mechanisms of cytomegalovirus-accelerated vascular disease: induction of paracrine factors that promote angiogenesis and wound healing. Curr Top Microbiol Immunol. 2008;325:397-415.

48. Caposio P, Orloff SL, Streblow DN. The role of cytomegalovirus in angiogenesis. Virus Res. 2011;157(2):204-211.

49. Sweeney MD, Ayyadurai S, Zlokovic BV. Pericytes of the neurovascular unit: key functions and signaling pathways. Nat Neurosci. 2016;19(6):771-783.

50. Rajkumar VS, et al. Platelet-derived growth factor-beta receptor activation is essential for fibroblast and pericyte recruitment during cutaneous wound healing. Am J Pathol. 2006;169(6):2254-2265.

51. Hellström M, Kalén M, Lindahl P, Abramsson A, Betsholtz C. Role of PDGF-B and PDGFR-beta in recruitment of vascular smooth muscle cells and pericytes during embryonic blood vessel formation in the mouse. Development. 1999;126(14):3047-3055.

52. Bergsten E, et al. PDGF-D is a specific, prote- ase-activated ligand for the PDGF beta-receptor. Nat Cell Biol. 2001;3(5):512-516.

53. Uutela M, et al. PDGF-D induces macrophage recruitment, increased interstitial pressure, and blood vessel maturation during angiogenesis. Blood. 2004;104(10):3198-3204.

54. Liu MY, Eyries M, Zhang C, Santiago FS, Khachigian LM. Inducible platelet-derived growth factor D-chain expression by angiotensin II and hydrogen peroxide involves transcriptional regulation by Ets-1 and Sp1. Blood. 2006;107(6):2322-2329.

55. Yurochko AD, Mayo MW, Poma EE, Baldwin AS, Huang ES. Induction of the transcription factor Sp1 during human cytomegalovirus infection mediates upregulation of the p65 and p105/p50 NF-kappaB promoters. J Virol. 1997;71(6):4638-4648.

56. Hadaczek P, et al. Cidofovir: a novel antitumor agent for glioblastoma. Clin Cancer Res. 2013;19(23):6473-6483.

57. De Schutter T, et al. Cidofovir treatment improves the pathology caused by the growth of human papillomavirus-positive cervical carcinoma xenografts in athymic nude mice. Cancer Lett. 2013;329(2):137-145.

58. Deutsch E, et al. Phase I trial evaluating the antiviral agent Cidofovir in combination with chemoradiation in cervical cancer patients. Oncotarget. 2016;7(18):25549-25557.

59. Snyder CM, Cho KS, Bonnett EL, Allan JE, Hill AB. Sustained CD8+ T cell memory inflation after infection with a single-cycle cytomegalovirus. PLoS Pathog. 2011;7(10):e1002295.

60. Ricklefs F, et al. Extracellular vesicles from highgrade glioma exchange diverse pro-oncogenic signals that maintain intratumoral heterogeneity. Cancer Res. 2016;76(10):2876-2881.

61. Muir ER. Preclinical arterial spin labeling measurement of cerebral blood flow. Methods Mol Biol. 2018;1718:59-70. 